\title{
A series of theoretical line profiles formed in the irradiated expanding atmospheres of close binary components ${ }^{\star}$
}

\author{
A. Peraiah and M. Srinivasa Rao \\ Indian Institute of Astrophysics, Koramangala, Bangalore-560034, India
}

Received March 20, 1997; accepted March 24, 1998

\begin{abstract}
We studied the effects of irradiation on the line formation in the expanding atmospheres of the components of close binary systems. We considered a two-level atom approximation in Non-LTE situation. The thickness of the atmosphere is assumed to be twice that of the stellar radius. Expansion velocities are assumed to be as large as 50 mean thermal units. We have computed the lines using total optical depths $10^{3}, 10^{4}$ and $10^{5}$ at the line centre. The irradiation from the secondary is assumed to be one, five and ten times the self radiation. The line fluxes in the line of sight are calculated by using the total source function which is the sum of the source functions due to self radiation and that due to irradiation. We notice that the expansion of the medium produces $P$ Cygni type profiles and the irradiation enhances the emission in the lines although the equivalent widths reduce considerably.
\end{abstract}

Key words: radiative transfer - lines: formation; profiles — binaries: close

\section{Introduction}

It is well known that when the two components of a binary system are sufficiently close to each other, their mutual irradiation is very important. The study of close binary stars is of great importance in stellar astrophysics. The atmospheres of the components of a close binary system are distorted mainly by two physical effects: (1) rotation of the component and (2) the tidal effect due to the presence of its companion. These effects make the atmospheres of these stars non-spherical. de Jager (1992) summarized the problem of non-spherical atmospheres and flows in outer

Send offprint requests to: A. Peraiah

e-mail: anna@iiap.ernet.in

* Figures 1(1-6), 2(1-30), 3(1-8), 4(1-8), 5(1-6) and 6(1-8) are available in electronic form at the http://www.edpsciences.com layers of young stars and other objects during the workshop at Baltimore. Non-sphericity changes the density distribution of the matter through which the radiation passes and as a consequence, the lines formed in such medium are modified. In addition to this, the presence of the secondary component's light falling on such a distorted components' atmosphere will affect the line profiles formed in these atmospheres. One also encounters the systematic mass motions in the atmospheres of these stars.

In the observational aspects of the problem Yu. Skulskij (1993) studied the variability of equivalent widths with phases and other characteristics of absorption and emission components of SiII $\lambda \lambda 6347,6371$ in the study of $\beta$ Lyre with CCD spectra. The variation of the absorption line equivalent widths depends on the orbital modulation and on the structure of the circumstellar gas in the close binary system. Fergusson \& James (1994) studied the eclipsing binary BE UMa for its reflection effect and as cataclysmic variable progenitor characteristics. The reflection effect is due to the relatively close proximity (about $\left.8 R_{\odot}\right)$ of a late type secondary of a very hot $T \sim 10^{5} \mathrm{~S}_{\mathrm{d}} \mathrm{O}$ star.

Parthasarathy et al. (1990) analyzed the ultraviolet spectrum (1175 A to $3200 \AA$ ) of the hydrogen-poor binary star HD 30353. The high resolution spectra show stellar wind profiles of NV, CIV, SiIV, CII, SiII, AlII, AlIII, MgII and FeII resonance lines. These profiles appear to shift towards the shorter side of the spectrum. They concluded that extended and multiple shells exist in the atmosphere with a source at temperature of $30000 \mathrm{~K}$, which could be a O-type or an early B-type star as suggested by the far UV flux distribution.

Theoretical studies about the reflection effect using actual model atmospheres are more recent. All the three possible combinations (i.e., when primary and secondary components are hot and hot, cool and cool, and hot and cool) are studied by Buerger $(1969,1972)$; Nordlund \& Vaz (1990); and Claret \& Gemenz (1992) respectively. Vaz (1985) and Wilson (1990) reviewed several aspects of reflection effect. They found that irradiation from the 
secondary component will effect the lines and as well as equivalent widths. They also found that the theoretical bolometric albedos have been found to be in good agreement with the observations. Peraiah \& Srinivasa Rao (1983) studied the effects of reflection on the formation of spectral lines in a purely scattering atmosphere and studied how the equivalent width changes due to irradiation from the secondary. However these calculations were done in static atmospheres.

The purpose of this study is to compute the spectral lines formed in the expanding atmospheres with light of the secondary falling on it. These atmospheres are distorted due to the combined effect of self rotation and tidal effect by the presence of the secondary component.

The transfer of radiation incident on the atmosphere of the component from the companion cannot be studied by using any symmetric solution of the equation of transfer. This needs a special treatment. We adopt angle-free one dimensional model (see Wing 1962; Sobolev 1963; Grant 1968). This procedure gives a fairly accurate solution provided we take large number of rays. The disadvantage in this technique is that either the angle dependence or frequency dependence cannot be incorporated.

Since it is difficult to handle asymmetric atmospheres in the radiative transfer calculations, we restrict our calculations to spherical geometry in this problem. As the gases in the atmospheres of close binaries move with large velocities, the problem can be treated in the comoving frame only. The solution of the radiative transfer equation in the comoving frame is given in Peraiah (1980). The radiation in the atmosphere consists of (1) self radiation of the component and (2) the incident radiation from the atmosphere of the companion. We need to treat the combination of these two radiation fields for calculations of the line profiles. The case of distorted atmosphere due to self rotation and tidal effect will be taken up in a subsequent paper.

\section{Brief description of the method of calculations}

We have assumed a spherical shape of the reflecting atmosphere to simplify the computational problems of radiative transfer. The geometry of the model is shown in Fig. 1.

Let $O$ and $O^{\prime}$ be the centres of the primary and the secondary respectively. The atmosphere of the primary is assumed to be spherical and divided into several discrete shells. We calculate the source functions of the radiation field emerging from the companion whose centre is at $O^{\prime}$ (see Fig. 1) and incident on the atmosphere of the component whose centre is at $O$. We consider the set of rays such as $S T P, E \tau P, O^{\prime} T_{1} P, E^{\prime} \tau^{\prime} P, W T_{2} P$ etc., emerging from the surface $S W$ of the companion and meeting at a point $P$ in the atmospheres of the component. These rays lie within the quadrilateral such as $P S O^{\prime} W$ and enter the boundary of the atmosphere of the component at points

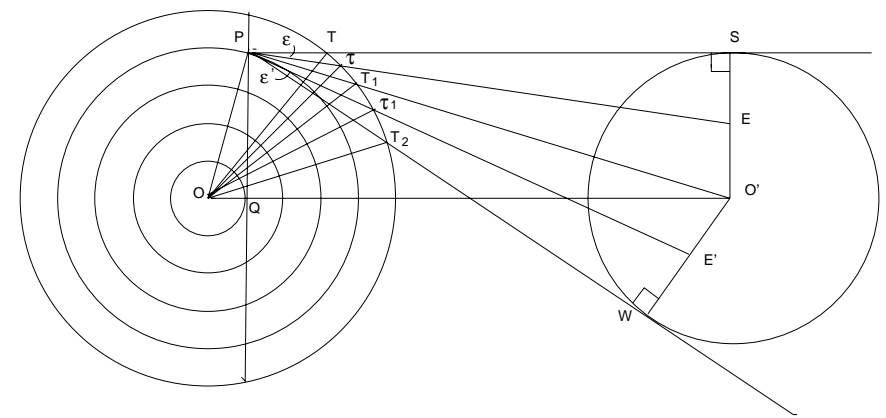

Fig. 1. Schematic diagram of the binary components with incident radiation from the surface of the secondary. $O$ and $O^{\prime}$ are the centres of gravity of the two components

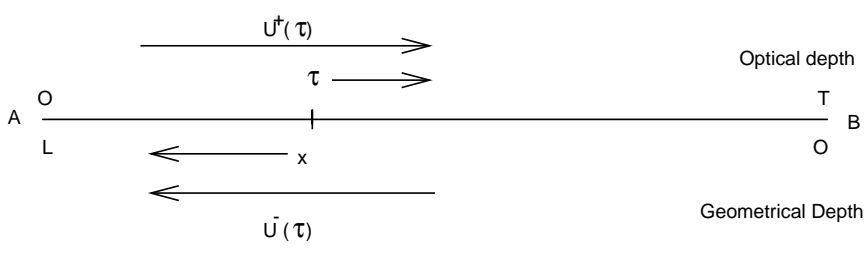

Fig. 2. Schematic diagram of the rod model

$T, \tau, T_{1}, \tau^{\prime}, T_{2}$ etc. The surfaces of the companion such as $S W$ will be different for different points $P$ in the atmosphere. The radiation field at $P$ is estimated by calculating the source function whose contribution comes from self radiation of the primary and that due to the incident radiation from the surface $S W$ of the secondary facing the primary. We need to estimate geometrical length of the ray segments such as $P \tau, P \tau^{\prime}$ etc, inside the atmosphere so that the transfer of radiation along these segments is estimated and its contribution to the source function at the point $P$ due to the incident radiation at $\tau, \tau^{\prime}$ etc. The length of the segments such as $P \tau$ in $S P O^{\prime}$ are given in the Appendix A (see Peraiah 1983 and Peraiah \& Rao 1983).

For a given density distribution we need to calculate the optical depth along the segments $P \tau, P \tau^{\prime}$ etc. The source function at points such as $P$ due to the irradiation are calculated using the one-dimensional transfer (Wing 1962; Sobolev 1963; Grant 1968). We will describe this procedure briefly below.

We consider a segment AB (see Fig 2) which has two rays oppositely directed to each other. The optical depth $\tau$ is given by,

$\tau=\tau(x)=-\int_{\mathrm{L}}^{x} \sigma\left(x^{\prime}\right) \mathrm{d} x^{\prime} ; \tau(0)=T$

where $\sigma\left(x^{\prime}\right)$ is the extinction coefficient and $T$ is the total optical depth. The optical depth is measured in the direction opposite to that of the geometrical segment.

We assume a steady state, monochromatic condition with local source function $B^{+}(\tau)$ in the direction of increasing $\tau$ and $B^{-}(\tau)$ in the reverse direction. $U^{+}(\tau)$ and $U^{-}(\tau)$ are the specific intensities in the $\tau$ increasing and decreasing directions respectively. 
The two equations of transfer for $U^{+}(\tau)$ and $U^{-}(\tau)$ are

$\frac{\mathrm{d} U^{+}}{\mathrm{d} \tau}+U^{+}=S_{1}^{+}$,

$\frac{\mathrm{d} U^{-}}{\mathrm{d} \tau}+U^{-}=S_{1}^{-}$,

where

$S_{1}^{+}=B^{+}(\tau)+\omega(\tau)\left[p(\tau) U^{+}(\tau)+\left(1-p(\tau) U^{-}(\tau)\right]\right.$,

$S_{1}^{-}=B^{-}(\tau)+\omega(\tau)\left[(1-p(\tau)) U^{+}(\tau)+p(\tau) U^{-}(\tau)\right]$,

are the source functions and $\omega(\tau)$ is the albedo for single scattering and the phase function $p=\frac{1}{2}$. The boundary conditions at $\tau=0$ and $\tau=T$ are given by

$U^{+}(\tau=0)=U_{1}$

$U^{-}(\tau=T)=U_{2}$,

where $U_{1}$ and $U_{2}$ will be specified later. The total source function $S_{\mathrm{d}}$ at any point $\tau$ is the combination of the scattered part of the local intensities $U^{ \pm}(\tau)$ in either directions and the diffuse radiation generated by the incident radiation at the boundaries $\tau=0$ and $\tau=T$ (the quantities $U_{1}$ and $U_{2}$ in Eqs. (6) and (7) respectively). This added to the local sources would give the total source function given by,

$S_{\mathrm{d}}^{+}(\tau)=S_{1}^{+}(\tau)+\omega(\tau)\left[p(\tau) U_{1} \mathrm{e}^{-\tau}+(1-p(\tau)) U_{2} \mathrm{e}^{-(T-\tau)}\right]$

$S_{\mathrm{d}}^{-}(\tau)=S_{1}^{-}(\tau)+\omega(\tau)\left[(1-p(\tau)) U_{1} \mathrm{e}^{-\tau}+p(\tau) U_{2} \mathrm{e}^{-(T-\tau)}\right]$

In this case, the boundary conditions are,

$U^{+}(0)=U^{-}(T)=0$.

We can write the Eqs. (2) and (3) as

$M \frac{\mathrm{d} U}{\mathrm{~d} \tau}+U=S_{1}$

where

$M=\left[\begin{array}{ll}1 & 0 \\ 0 & -1\end{array}\right], U=\left[\begin{array}{l}U^{+} \\ U^{-}\end{array}\right], S_{1}=\left[\begin{array}{l}S_{1}^{+} \\ S_{1}^{-}\end{array}\right]$,

and Eqs. (4) and (5) and (8) and (9) will be written as,

$S_{1}(\tau)=B(\tau)+\omega(\tau) P(\tau) U(\tau)$,

$S_{\mathrm{d}}(\tau)=S_{1}(\tau)+\omega(\tau) P(\tau) U_{\mathrm{b}}(\tau)$,

where

$B=\left[\begin{array}{l}B^{+} \\ B^{-}\end{array}\right], P=\left[\begin{array}{ll}p & 1-p \\ 1-p & p\end{array}\right]$,

$U_{\mathrm{b}}(\tau)=\left[\begin{array}{ll}U_{1} & \mathrm{e}^{-\tau} \\ U_{2} & \mathrm{e}^{-(T-\tau)}\end{array}\right]$.

The mathematical aspects of the solution of the Eqs. (2) and (3) are discussed in Sobolev (1963) and Grant (1968). We shall merely quote the relevant results and these are given in Appendix B.
Using equations (B1) and (B2) one can calculate the source function at points such as $P$, due to the incident radiation from the secondary component.

Now, we need to estimate the source function due to self radiation of the component. This can be done by solving the line transfer equation for a Non-LTE two-level atom in the comoving frame in spherical symmetry. This is given by (see Peraiah 1984; Peraiah et al. 1987)

$$
\begin{aligned}
& \mu \frac{\partial I(x, \mu, r)}{\partial r}+\frac{\left(1-\mu^{2}\right)}{r} \frac{\partial I(x, \mu, r)}{\partial \mu}=K(x, r) S_{\mathrm{L}}(r) \\
& +K_{\mathrm{c}}(r) S_{\mathrm{c}}(r)-\left[K(x, r)+K_{\mathrm{c}}(r)\right] I(x, \mu, r) \\
& +\left[\left(1-\mu^{2}\right) \frac{V(r)}{r}+\mu^{2} \frac{\mathrm{d} V(r)}{\mathrm{d} r}\right] \frac{\partial I(x, \mu, r)}{\partial x},
\end{aligned}
$$

and

$$
\begin{aligned}
& -\mu \frac{\partial I(x,-\mu, r)}{\partial r}-\frac{\left(1-\mu^{2}\right)}{r} \frac{\partial I(x,-\mu, r)}{\partial \mu}= \\
& K(x, r) S_{\mathrm{L}}(r)+K_{\mathrm{c}}(r) S_{\mathrm{c}}(r)-[K(x, r) \\
& \left.+K_{\mathrm{c}}(r)\right] I(x,-\mu, r)+\left[\left(1-\mu^{2}\right) \frac{V(r)}{r}\right. \\
& \left.+\mu^{2} \frac{\mathrm{d} V(r)}{\mathrm{d} r}\right] \frac{\partial I(x,-\mu, r)}{\partial x},
\end{aligned}
$$

where $I(x, \pm \mu, r)$ is the specific intensity of the ray at an angle $\cos ^{-1} \mu[\mu \epsilon(0,1)]$ with the radius vector at the radial point $r$ with frequency $x\left(=\left(\nu-\nu_{0}\right) / \Delta \nu_{\mathrm{D}}\right.$ where $\nu_{0}$ and $\nu$ are the frequency points at the line centre and at any point in the line and $\Delta \nu_{\mathrm{D}}$ is the standard frequency interval such as Doppler width) $V(r)$ is the velocity of the gas at $r$ in units of mean thermal units (mtu) and $K(x, r)$ and $K_{\mathrm{c}}(r)$ are the absorption coefficients per unit frequency interval in the line and the continuum respectively. The quantities $S_{\mathrm{L}}$ and $S_{\mathrm{c}}$ are the line and continuum source functions given by,

$S_{\mathrm{L}}(r)=(1-\epsilon) \int_{-\infty}^{+\infty} J(x, r) \phi(x) \mathrm{d} x+\epsilon B(x, T(r))$,

$S_{\mathrm{c}}(r)=\rho(r) B(x, T(r))$,

$K(x, r)=K_{\mathrm{L}}(r) \phi(x)$,

where $K_{\mathrm{L}}(r)$ is the line-centre absorption coefficient and $\phi(x)$ is the normalized line profile and $\rho(r)$ is an arbitrary factor less than one and $B(x, T(r))$ is the Planck function with frequency $x$, and temperature $T$ at the radial point $r . J(x, r)$ is the mean intensity given by,

$J(x, r)=\frac{1}{2} \int_{-1}^{+1} I(x, r, \mu) \mathrm{d} \mu$.

The quantity $\epsilon$ is the probability per scattering that a photon is thermalised by collisional de-excitation of the excited states, and this is given by,

$\epsilon=C_{21}\left[C_{21}+A_{21}\left[1-\exp \left(-h \nu_{0} / k T\right)\right]\right]^{-1}$ 
where $C_{21}$ is the collisional transition rate from level 2 to 1 and $A_{21}$ is the Einstein spontaneous emission probability for transition from level 2 to 1 . The quantities $h$ and $k$ are the Planck constant and Boltzmann constant respectively. From Eqs. (17) to (22), we obtain the source function due to self radiation, given by

$S_{\mathrm{S}}(x, r)=\frac{\phi(x)}{\beta+\phi(x)} S_{\mathrm{L}}(r)+\frac{\beta}{\beta+\phi(x)} S_{\mathrm{c}}(r)$.

where $\beta$ is the ratio of absorption coefficients in the continuum and line centre.

Finally we calculate the total source function by adding $S_{\mathrm{d}}(\tau)$ in Eq. (14) and $S_{\mathrm{s}}(x, r)$ in Eq. (24) and obtain,

$S=S_{\mathrm{s}}+S_{\mathrm{d}}$.

We calculate the set of source functions at the points of intersection of the ray parallel to the line of sight and the shell boundaries. These source functions are used to calculate the emergent specific intensities at infinity (or at the observer's point), by using the formula (see Peraiah \& Srinivasa Rao 1983)

$I_{n+1}(r)=I_{0}(n) \mathrm{e}^{-\tau}+\int_{o}^{\tau} S(t) \mathrm{e}^{-[-(\tau-t)]} \mathrm{d} t$,

where $I_{n}(r)$ corresponds to the specific intensity of the ray passing through shell numbers $n$ and $n+1$ and corresponding to the perpendicular to the axis $O O^{\prime}$ at different radii. $I_{0}(n)$ corresponds to the incident intensity at the boundary of the shell and $\tau$ is the optical depth in the sector along the ray path. The source function $S(t)$ is calculated by linear interpolation between $S\left(t_{n}\right)$ and $S\left(t_{n+1}\right)$. The specific intensity at the boundary of each shell is calculated by using Eq. (26).

The atmosphere in question is divided in to $n$ shells (see Fig. 1) where $n=1$ corresponds $\tau=T$ and $n=100$ corresponding to $\tau=0$, and $\tau$ is the optical depth at any point and $T$ is the total optical depth. The total optical depth is set in advance. The incident radiation at $Q$, the bottom of the atmosphere, (see Fig. 1) is given as

$I_{\mathrm{s}}\left(\tau=T, \mu_{j}\right)=1$.

The incident radiation from the secondary is given in terms of $I_{\mathrm{S}}$ in the ratio $I$, where $I$ is given by

$I=\frac{U_{1}}{I_{\mathrm{s}}}$.

The velocities of expansion of the gas are expressed in terms of mean thermal units $V_{T}(\mathrm{mtu})$ given by,

$V_{T}=\left[\frac{2 k T}{m_{i}}\right]^{\frac{1}{2}}$

where $k$ is the Boltzmann constant and $T$ is the temperature and $m_{i}$ is the mass of the ion. The velocity at $n=1$ or $\tau=T$ is $V_{A}$ and $V_{B}$ is the velocity at $n=100$ or $\tau=0$ are given in terms of mtu or $V_{T}$ as

$V_{A}=\frac{v_{A}}{V_{T}}$
$V_{B}=\frac{v_{B}}{V_{T}}$

where $v_{A}$ and $v_{B}$ are the velocities at the inner radius $A$, outer radius $B$ at radial point $r$ respectively in units of mtu of gas. We assume uniform expansion of the gases for the sake of simplicity. The proximity of the component is measured in terms of separation parameter $r_{1} / R$, where $r_{1}$ is the radius of the primary and $R$ is the separation of centres of gravity of the two components. The ratio of the outer to the inner radii $(B / A)$ of the atmosphere is always taken to be 2 . The actual thickness in the components could be much larger than what we have considered here. As the number of parameters is large, we restricted our calculations to this modest thickness of the atmosphere.

The variation of the source functions are shown against the shell numbers ( $n=1$ to 100) for different parameters are shown. The line profile fluxes $\left(F_{Q} / F_{\mathrm{c}}\right)$ are plotted against the normalized frequency $Q$, where

$Q=x / x_{\max }$,

$F_{Q}=F\left(x_{Q}\right)$,

$F_{C}=F\left(x_{\max }\right)$,

and

$x=\left(\nu-\nu_{0}\right) / \Delta \nu_{D}$,

$x_{\max }=|x|+V_{B}$,

$\Delta \nu_{D}=\nu_{0} \frac{v_{T}}{c}$

$x$ lies between \pm 5 units. The equivalent widths are calculated by the relation,

$E Q . W=\int_{x_{\min }}^{x_{\max }}\left(1-F_{Q} / F_{\mathrm{c}}\right) \mathrm{d} x$

where

$x_{\min }=-\left(|x|+V_{B}\right)$.

\section{Results and discussion}

The equations of line transfer given in the Eqs. (17) and (18) are solved following the procedure described in Peraiah et al. (1987). The optical depths along these segments $P \tau P \tau^{\prime}$ are calculated using the Eq. (1). We set $\sigma$ as the electron scattering coefficient equal to (Thomson cross section) to $6.652510^{-25} \mathrm{~cm}^{2}$. The lengths of the segments change between 0 and $2 r$ where $r$ is the radius of the component. We have set an electron density of $10^{14} \mathrm{~cm}^{-3}$. The maximum optical depth is 97.5 while optical depth of the segment along the $x$-axis $O O^{\prime}$ is 66.525 where the radius of the star is taken to be $10^{12} \mathrm{~cm}$ and the thickness of the atmosphere as $10^{12} \mathrm{~cm}$. The parameters that are used in the calculations are listed below.

$B / A=$ ratio of the outer to the inner radii of the atmosphere of the primary component and whose reflection effect is being studied $(=2) \cdot n=$ number of shells into which the atmosphere of the component is divided, 
$r_{1} / R=$ ratio of the radius of the component to that of the line joining the centres of gravity of the two components $r_{1}=210^{12} \mathrm{~cm}$.

$V_{A}=$ initial velocity of expansion in units of mtu at $n=1$ (see Eqs. (29) and (30)).

$V_{B}=$ final velocity in units of mtu at $n=100$ (see Eq. $(31))$.

$S=$ total source function (see Eq. (25)),

$S_{\mathrm{s}}=$ source function due to self radiation (see Eq. (24)),

$I=$ ratio of incident radiation to that of self radiation of the star (see Eq. (28)),

$\epsilon=$ probability per scatter that a photon is thermalised by collisional de-excitation (see Eq. (23)),

$\beta=$ ratio of absorption coefficient in continuum to that in the line,

$T=$ total optical depth,

$Q=x / x_{\max }$ (see Eq. (32)),

$F_{Q} / F_{\mathrm{c}}=$ ratio of the line flux at the normalized frequency $Q$ to that in the continuum or at $x_{\max }$ (see Eqs. (33) and $(34))$,

$(R ; N . R)=$ with reflection and with no reflection,

$H_{\mathrm{e}} / H_{\mathrm{a}}=$ height of the emission to the depth of absorption in the line,

$T_{\mathrm{e}}=$ temperature in the atmosphere.

Few results are presented in Figs. 3, 4, and 5 for different parameters. The figures are self explanatory as far as the parameters are concerned. The atmosphere of the primary whose centre is at $O$ is divided into 100 shells (see Fig. 1). The separation of the components as $r_{1} / R$ where $r_{1}$ is the radius of the primary and $R\left(=O O^{\prime}\right)$ is the separation of the centres of gravity of the components. We have considered two cases of separation $r_{1} / R=1 / 2$ and $1 / 5$ and the atmospheric extension is set to equal to stellar radius or $B / A=2$. The total radial optical depth $T$ is taken to be $10^{3}, 10^{4}$ and $10^{5}$. The velocities of expansion are measured in terms of mean thermal units and uniform expansion velocity law is assumed. If $V_{A}$ and $V_{B}$ are the velocities at $A$ and $B$ respectively, then the velocity at any shell boundary $V_{n}=\left[V_{A}+\frac{V_{B}-V_{A}}{N}\right]$. At $A\left(\tau=\tau_{\max }=T\right)$ the velocity is $V_{A}$ and at $B(\tau=0)$ the velocity is $V_{B}$. The parameters $\epsilon$ which is defined in Eq. (23) is the probability that a photon is destroyed by collisional de-excitation and it is $<1$ for non-LTE line formation and this is set to equal to 0 and $10^{-4}$. The quantity $\beta$ which is the ratio of absorption in the continuum to that in the line centre is set to 0 and $10^{-4}$ in our calculations.

Figure 3a gives the source functions $S_{\mathrm{s}}$ and $S$ given in Eqs. (24) and (25) for various parameters shown in the figure, across the atmosphere from $n=1$ to $n=100$. These results represent a static and scattering medium with $V_{A}=V_{B}=0$ and $\epsilon=\beta=0$ and the incidence radiation factor $I=1$ (see Eq. (28)). The incident radiation at $A$ is given according to Eq. (27). The source function $S_{\mathrm{s}}$ (which does not contain the reflected radiation) decreases slowly from the point $\tau=T$ to the point $\tau=0$ in the scattering medium with $T=10^{4}$. When the reflected radiation is included, the source functions for $r_{1} / R=1 / 2$ and $1 / 5$ are considerably enhanced as these source functions include the incident radiation from the companion along the axis $O O^{\prime}$. Figure $3 \mathrm{~b}$ gives the line profile in the direction of the line of sight, corresponding to the source functions given in Fig. 3a. The line fluxes are plotted against the normalized frequency points $Q$ (see Eq. (32)). As the medium is static, the profile are symmetric with central absorption. More photons are removed from the centre when the line central optical depth is $10^{4}$, and the centre becomes almost black. When the incident radiation from the component is added, there is more emission in the central portion of the line. The shapes of the lines in all these cases remain symmetric about the centre of the line. Figure $3 \mathrm{c}$ gives the variation of the same quantities as those given in Fig. 3a except that velocity $V_{B}=50$ mean thermal units. There is a marked difference in the variation of the source functions in the two cases when $V_{B}=0$ in Fig. 3a and $V_{B}=50 \mathrm{mtu}$ in Fig. 3c. There is a sudden fall in the source functions near $\tau=T$ and these remain almost constant throughout the rest of the atmosphere. Figure $3 d$ presents the line profiles along the line of sight corresponding to the source functions presented in Fig. 3c. These are similar to P-Cygni type profiles formed in an expanding media with blue shifted absorption. However the emission although small, confines more or less to the centre of the line formed in static medium. The reason for this is that the absorption core is formed in the portion of the atmosphere which is directly in between the star and the observer. As it is moving towards the observer there will be a Doppler shift of the frequencies of the line photons towards the blue side of the centre of the line. The photons that are emitted in the side lobes of the atmosphere are merely scattered and the Doppler effect due to the velocities in the farther part and nearer part (with respect to the observer's point) will nearly counter each other, maintaining an approximate symmetric emission about the centre. Therefore the asymmetry caused by the Doppler shifts is minimal in the emission part of the line. Figure 3e gives the variation of equivalent widths against the expanding velocities $V_{B}$ for the parameters shown in Fig. 3. We can see that when no radiation is incident from the companion the equivalent widths are much larger than when there is incident light falling on the component from the companion. This can be understood from the fact that more photons are emitted through the line when external radiation is falling on the atmosphere from out side, which is also clear from a comparison of profiles given in Figs. 3b and $3 \mathrm{~d}$. However in both the cases of reflection and no reflection the equivalent widths increase with the increasing velocities of expansion. Figure $3 \mathrm{f}$ gives the variation of the ratio of height of emission $\left(H_{\mathrm{e}}\right)$ to that of depth absorption $\left(H_{\mathrm{a}}\right)$. There is no change in the reflection and non reflection cases. However it is noteworthy that this ratio 

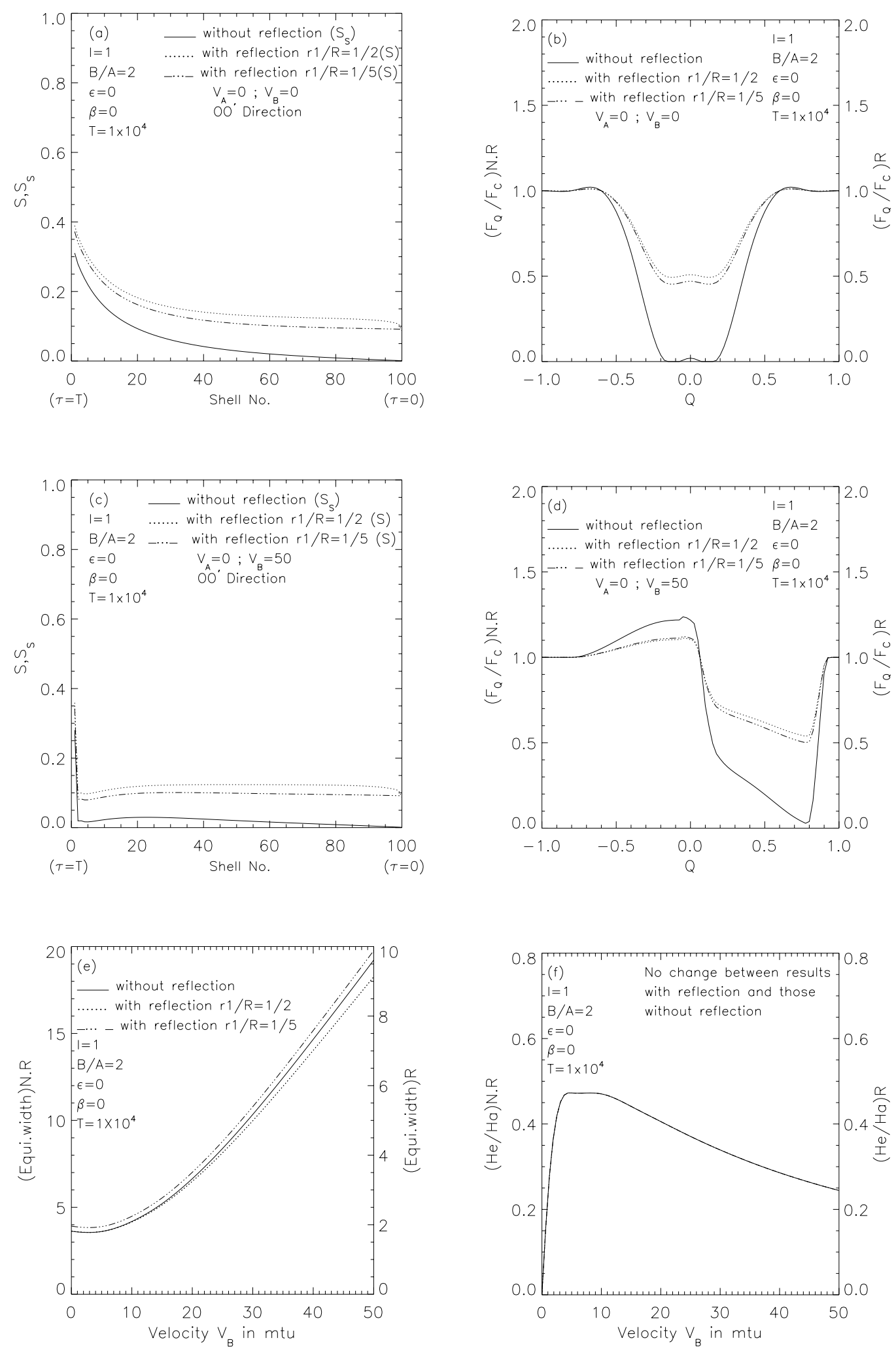

Fig. 3. a) The source functions $S$ and $S_{\mathrm{s}}$ are shown with respect to the shell numbers in a scattering medium for velocity $V_{A}=0 ; V_{B}=0$. b) Line profiles with reflection and without reflection from the secondary components for a static and scattering medium with total optical depth $T=10^{4}$ and $V_{A}=0 ; V_{B}=0$. c) The source functions $S$ and $S_{\text {s }}$ are shown with respect to the shell numbers in a scattering medium for velocity $V_{A}=0 ; V_{B}=50$. d) Line profiles with reflection and without reflection from the secondary components for a static and scattering medium with total optical depth $T=10^{4}$ and $V_{A}=0 ; V_{B}=50$. e) Equivalent widths of the lines are plotted against the expansion velocity $V_{B}$. f) The ratios of the height of the emission to the depth of the absorption in the lines for both the case of reflected radiation and non reflected radiation are shown against the velocity of expansion $V_{B}$ 

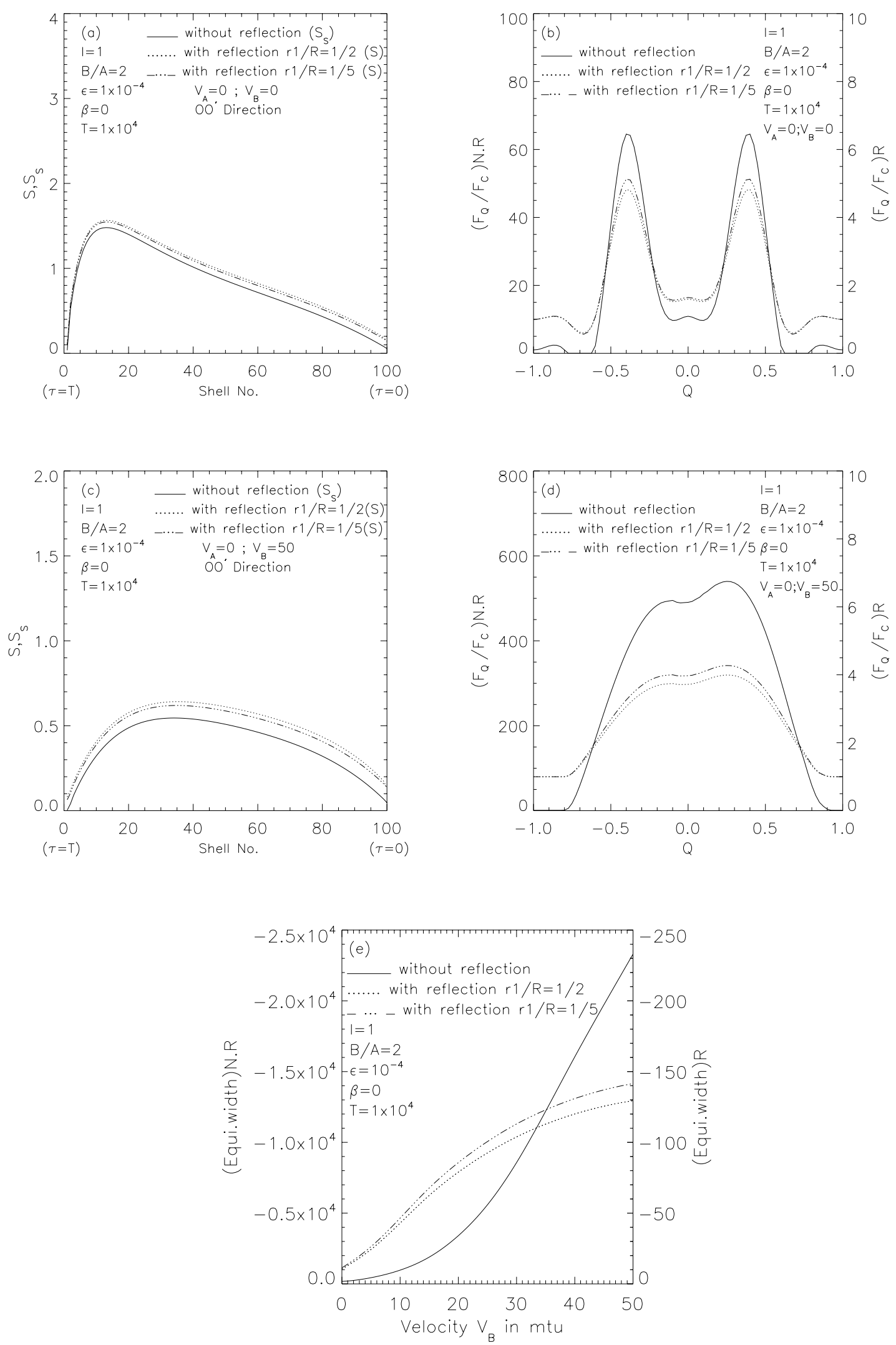

Fig. 4. a) Same as those given in Fig. 3a but with $\epsilon=110^{-4}$ b) Same as those given in Fig. 3b but with $\epsilon=110^{-4}$ c) Same as those given in Fig. 3c but with $\epsilon=110^{-4}$ d) Same as those given in Fig. 3d but with $\epsilon=110^{-4}$ e) Same as those given in Fig. 3e but with $\epsilon=110^{-4}$ 

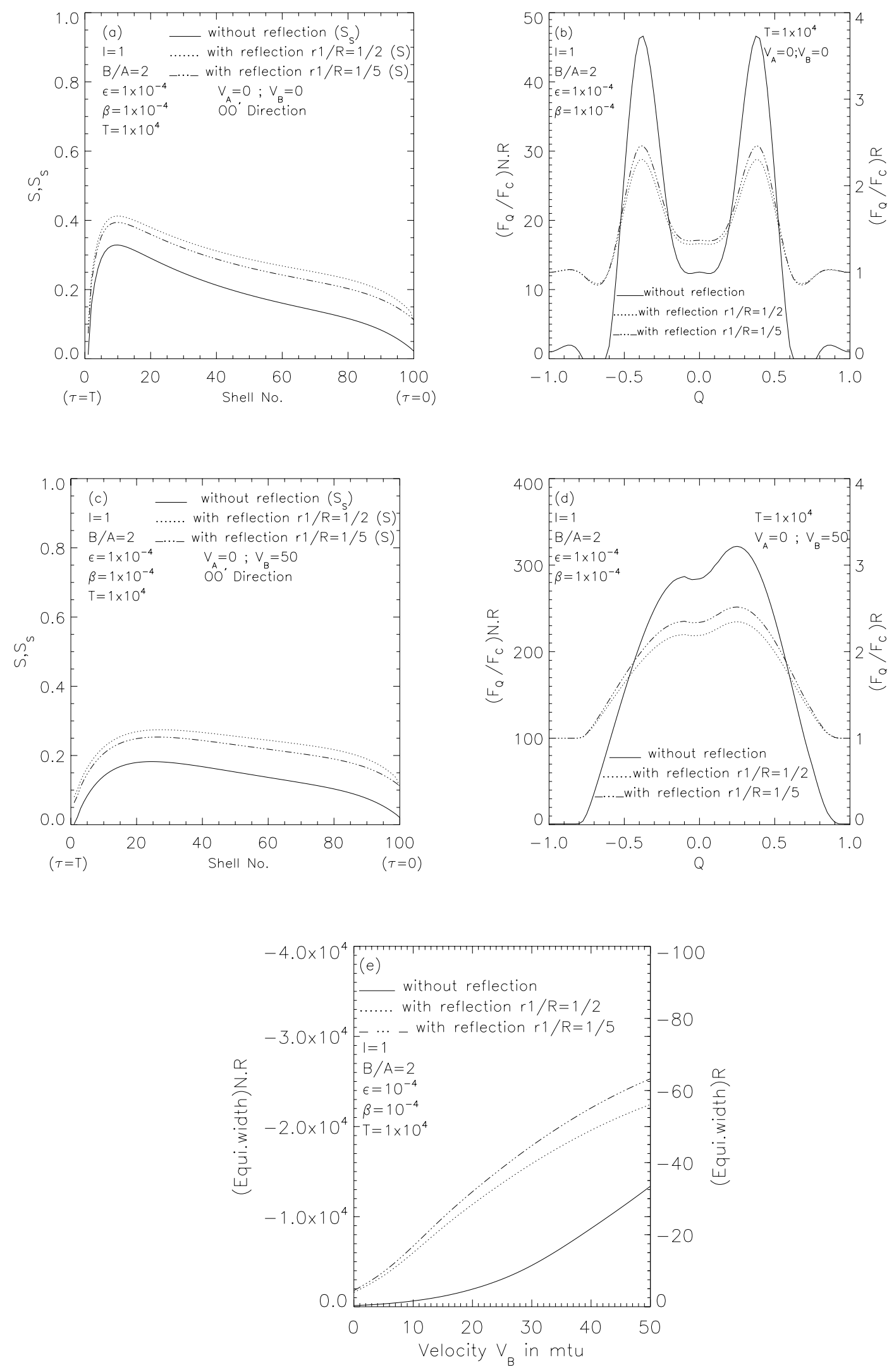

Fig. 5. a) Same as those given in Fig. 3a but with $\epsilon=110^{-4}$ b) Same as those given in Fig. 3b but with $\epsilon=110^{-4}$ c) Same as those given in Fig. 3c but with $\epsilon=110^{-4}$ d) Same as those given in Fig. 3d but with $\epsilon=110^{-4}$ e) Same as those given in Fig. 3e but with $\epsilon=110^{-4}$ 
reaches a maximum at about $V_{B}=4$ to $10 \mathrm{mtu}$ and then falls slowly as the expansion velocities increase.

Figures $4 \mathrm{a}$ to $4 \mathrm{e}$ gives the results for the set $\epsilon=10^{-4}$ and $\beta=0$. The Planckian $B(T(r))$ is set to equal to 1 uniformly throughout the medium. The source functions described in Fig. 4a represent the internal emission of photons. Although we have given a uniform emission $B(T(r))=1$ throughout more of the radiation is scattered towards the boundary $\tau=0\left(r=r_{\max }=B\right)$ this is the effect of sphericity or curvature scattering. The incident radiation increases the source function only marginally. Corresponding line profiles (along the line of sight) are given in Fig. 4b. these profiles show emission with absorption at line centre and these are symmetric with respect to the centre of the line as the medium treated here is static. Figure 4c gives the source functions of a medium which is expanding with $V_{B}=50 \mathrm{mtu}$. These are different from those given in Fig. 4a for a static medium. The maximum is spread over a larger spatial extent which is the effect of scattering in an expanding gas. The corresponding line profiles are given in Fig. 4d. The emission is spread through the line except for a small absorption around the central position of the line. The equivalent widths of those lines are plotted in Fig. 4e with respect to expansion velocities $V_{B}^{\prime}$ s. It is interesting to note that when there is no reflection, the equivalent widths of the emission lines increase with velocities of expansion while the incident radiation from outside reduces the emission line equivalent widths considerably.

In Figs. 5a to 5e, the results of the cases $\epsilon=\beta=10^{-4}$ are plotted. These results show similar characteristics those given in Figs. 4a to 4e.

We have performed several calculations for different parameters to study the effects of irradiation on line formation in the expanding atmosphere of the component of a close binary system. We have studied the variation of source functions, with different velocity gradients and also various values of irradiation from the secondary component. The line profiles computed with reflection are compared with those computed without reflection and for several cases of the proximity of the two components. We obtained P-Cygni type profiles. These results are described in six sets. The figures are self explanatory as far as the parameters that are used, are concerned.

\section{Appendix A:}

The segment $P \tau$ in $S P O^{\prime}$ is given as (see Fig. 1)

$P \tau=O P\left[A\left(B^{\prime} / B\right)+\left(1-A^{2}\right)^{1 / 2}\right]$,

where

$B=(O P / O T) A, B^{\prime}=\left(1-B^{2}\right)^{1 / 2}$,

$A=\xi \eta^{\prime}-\xi^{\prime} \eta$

$\eta=\sin E=S E / P E, \eta^{\prime}=\cos E$, $\xi=a b c+a^{\prime} b^{\prime} c+a^{\prime} b c^{\prime}-a b^{\prime} c^{\prime}$,

$\xi^{\prime}=\left(1-\xi^{2}\right)^{1 / 2}$.

And

$a=O Q / O P, a^{\prime}=\left(1-a^{2}\right)^{1 / 2}$,

$b=P S / O^{\prime} P, b^{\prime}=\left(1-b^{2}\right)^{1 / 2}$,

$c=P Q / O^{\prime} P, C^{\prime}=\left(1-c^{2}\right)^{1 / 2}$.

Similarly the segments such as $P \tau^{\prime}$ in $O^{\prime} P W$ are given by,

$P \tau^{\prime}=O P\left[\mu\left(s^{\prime} / s\right)+\left(1-\mu^{2}\right)\right]$,

where

$\mu=\nu^{\prime} \Delta+\Delta^{\prime} \nu$

$s=(O P / O T) \mu, s^{\prime}=\left(1-s^{2}\right)^{1 / 2}$,

$\nu=W E^{\prime} / P E^{\prime}, \nu^{\prime}=\left(1-\nu^{2}\right)^{1 / 2}$,

$P E^{\prime 2}=P W^{2}+W E^{\prime 2}, \Delta=\xi\left(1-2 \delta^{2}\right)-2 \xi^{\prime} \delta \delta^{\prime}$,

$\Delta^{\prime}=\left(1-\Delta^{2}\right)^{1 / 2}, \delta=S O^{\prime} / P O^{\prime}, \delta^{\prime}=\left(1-\delta^{2}\right)^{1 / 2}$.

\section{Appendix B:}

As we are dealing with scattering along the ray and the medium has no sources, we can set $B=0$ and $U_{2}=0$. Then the intensities $\tau$ are given by,

$U^{+}(\tau)=U_{1} \mathrm{e}^{-k \tau} \frac{1-r^{2} \mathrm{e}^{-2 k(T-\tau)}}{1-r^{2} \mathrm{e}^{-2 k t}}$,

$U^{-}(\tau)=r U_{1} \frac{\mathrm{e}^{-k \tau}-\mathrm{e}^{-k(2 T-\tau)}}{1-r^{2} \mathrm{e}^{-2 k T}}$,

where

$k^{2}=(1-\omega)[1+\omega(1-2 p)]$,

and

$$
r=\frac{k-1+\omega}{k+1-\omega}
$$

And the emergent intensities are

$U^{+}(T)=U_{1} \frac{\mathrm{e}^{-k T}\left(1-r^{2}\right)}{1-r^{2} \mathrm{e}^{-2 k T}}$,

$U^{-}(0)=U_{1} r \frac{1-\mathrm{e}^{-2 k T}}{1-r^{2} \mathrm{e}^{-2 k T}}$.

If we represent the reflection and transmission coefficients by $r(T)$ and $t(T)$ respectively, then and

$r(T)=r \frac{1-\mathrm{e}^{-2 k T}}{1-r^{2} \mathrm{e}^{-2 k T}}$,

$t(T)=\frac{\left(1-r^{2}\right) \mathrm{e}^{-k T}}{1-r^{2} \mathrm{e}^{-2 k T}}$.

We set $p=\frac{1}{2}$ (for isotropic scattering) then

$k=(1-\omega)^{1 / 2}, r=\frac{1-k}{1+k}$.

In the above treatment we assumed that $\omega<1$. If $\omega=1$, the case of pure scattering, the treatment will be different and we obtain, (for $B=0$ and $U_{2}=0$ )

$\mathbf{U}=\frac{U_{1}}{1+T(1-p)}\left[\begin{array}{l}1+(T-\tau)(1-p) \\ (T-\tau)(1-p)\end{array}\right]$ 
The reflection and transmission factors are

$r(T)=\frac{T(1-p)}{1+T(1-p)} \rightarrow 1$ as $T \rightarrow \infty$,

$t(T)=\frac{1}{1+T(1-p)} \rightarrow 0$ as $T \rightarrow \infty$,

so that

$r(T)+t(T)=1$.

which express conservation of energy.

\section{References}

Buerger P., 1969, ApJ 158, 1151

Buerger P., 1972, ApJ 177, 657

Claret, Gemenz, 1992, A\&A 256, 572

de Jager C., 1992, Workshop on Non-isotropic and variable outflows from stars, Baltimore, Maryland, MD (U.S.A.)

Drissen L., Leitherer C., Nota A. (eds.) Astron. Soc.

Pac. Conf. Ser. 22, Astronomical Society of Pacific, San Francisco CA (U.S.A.) p. 400
Ferguson D.H., James T.A., 1994, ApJS 94, 723

Grant I.P., 1968, Lecture Notes on New Methods in Radiative Transfer

Nordlund A., Vaz L.P.R., 1990, A\&A 228, 231

Parthasarathy M., Hack M., Tektunali G., 1990, A\&A 230, 136

Peraiah A., 1980, Acta Astron. 30, No. 4, 525

Peraiah A., 1983, J. Astrophys. Astron. 4, 151

Peraiah A., 1984, from "Methods in Radiative Transfer" Kalkofen W. (ed.). Cambridge University Press

Peraiah A., Srinivasa Rao M., 1983, J. Astrophys. Astron. 4, 175

Peraiah A., Varghese B.A., Srinivasa Rao M., 1987, A\&AS 69, 345

Sobolev V.V., 1963, A Treatise on Radiative Transfer Van Nostrand, New York

Vaz L.P.R., 1985, Ap\&SS 113, 349

Vaz L.P.R., Nordlund A., 1985, A\&A 147, 281

Wilson R.E., 1990, ApJ 356, 613

Wing G.M., 1962, An introduction to Transfer Theory John Wiley, New York

Yu. Skul'skij M., 1993, Soviet Astron. Lett. 19, No. 1, Translated from Pis'ma Astron. Zh., Tom 19, No. 1, p. 45 